\title{
NON-GAUSSIAN FEATURES OF LINEAR COSMIC STRING MODELS
}

\author{
P. P. Avelino \\ Centro de Astrofisica, Universidade do Porto, Rua do Campo Alegre 823, 4150 Porto, Portugal \\ E. P. S. Shellard and J. H. P. Wu \\ Department of Applied Mathematics and Theoretical Physics, University of Cambridge, \\ Silver Street, Cambridge CB3 9EW, UK \\ AND \\ B. Allen \\ Department of Physics, University of Wisconsin-Milwaukee, P.O. Box 413, Milwaukee, WI 53201 \\ Received 1998 April 9; accepted 1998 August 21; published 1998 September 24
}

\begin{abstract}
We investigate the non-Gaussian properties of cosmic string-seeded linear density perturbations with cold and hot dark matter backgrounds using high-resolution numerical simulations. We compute the one-point probability density function of the resulting density field and its skewness, kurtosis, and genus curves for different smoothing scales. A semianalytic model is then invoked to provide a physical interpretation of our results. We conclude that on scales smaller than $1.5\left(\Omega h^{2}\right)^{-1} \mathrm{Mpc}$, perturbations seeded by cosmic strings are very non-Gaussian. These scales may still be in a linear or mildly nonlinear regime in an open or $\Lambda$ universe with $\Gamma=\Omega h \lesssim 0.2$.
\end{abstract}

Subject headings: cosmic strings — dark matter — galaxies: clusters: general -

large-scale structure of universe

\section{INTRODUCTION}

At present, the two main candidates for the origin of cosmic structure are inflation and topological defects (for a review, see Vilenkin \& Shellard 1994). Although both scenarios may produce a power spectrum of density perturbations that is consistent with observations, they have very different predictions regarding the statistical properties of the density field. While most inflationary models produce Gaussian random-phase initial conditions, defect models produce non-Gaussian perturbations, particularly on small scales. New results from cosmic string-seeded structure formation using high-resolution simulations (Avelino et al. 1998a, 1998b) were encouraging for models with $\Gamma=\Omega h=0.1-0.2$ (see also Battye, Robinson, \& Albrecht 1997); both the mass fluctuation amplitude at $8 \mathrm{~h}^{-1}$ $\mathrm{Mpc}, \sigma_{8}$, and the power spectrum shape of cosmic string-induced cold dark matter fluctuations $P(k)$ were consistent within uncertainties with observational data (Peacock \& Dodds 1994; Viana \& Liddle 1996). However, because cosmic strings induce non-Gaussian density perturbations on small scales, the power spectrum alone is insufficient to describe all of the statistical properties of such a density field. This is even more important in open or $\Lambda$ models because in those models the characteristic scales of the density field are shifted to larger scales relative to a flat model with $\Lambda=0$.

In this Letter, we investigate the non-Gaussian properties of the linear density field induced by cosmic strings using higher order statistics such as the skewness and the kurtosis of a onepoint probability density function (PDF), as well as genus statistics. The non-Gaussian properties we reveal provide a significant observational signature for cosmic string-seeded structure formation models on small length scales. We note that previous analytic work has investigated the string-induced velocity field on scales above several $h^{-1} \mathrm{Mpc}$, which was inferred to be Gaussian (Vachaspati 1992; Moessner 1995) and that some of the features we study here were also observed in global topological defect models, notably for textures (Park, Spergel, \& Turok 1991). Past work on genus statistics in the context of topological defects was made using toy models that incorpo- rated some important features of the models in question (Brandenberger, Kaplan, \& Ramsey 1993; Albrecht \& Robinson 1995; Avelino 1997).

Our first step in the present analysis was to perform highresolution numerical simulations of cosmic string networks in an expanding universe (Allen \& Shellard 1990) from which we subsequently computed the causally sourced density field with either a cold (CDM) or hot dark matter (HDM) background. The cosmic string simulations had a dynamic range extending from before the radiation-matter transition at $0.4 \eta_{\mathrm{eq}}$ through to deep into the matter era $8.4 \eta_{\mathrm{eq}}$, where $\eta_{\mathrm{eq}}$ is the conformal time at radiation-matter density equality. The structure formation simulation boxes contained $256^{3}$ grid points and their physical volume was in the range $\left(4-100 h^{-1} \mathrm{Mpc}\right)^{3}$. A much more detailed description of these methods is given by Avelino et al. (1998a, 1998b).

\section{NON-GAUSSIAN TEST STATISTICS}

We first convolved the density field obtained above with a top-hat window at different smoothing scales $R$ and then calculated the resulting PDF $p(\nu)$, where $\nu$ is the number of standard deviations from the mean. The chosen smoothing scales were always at least 3 times larger than the grid spacing to guarantee a sufficient resolution for the smoothing and at least 12 times smaller than the box size to reduce sample variance effects. We then used the skewness and the kurtosis to test their Gaussianity. The skewness is defined as $M_{3} / M_{2}^{3 / 2}$, where $M_{i}$ is the $i$ th central moment of a PDF. Its sign indicates the skewed direction against a normal distribution. The kurtosis is defined as $M_{4} / M_{2}^{2}-3$ and measures the size of the side tails against those of a normal distribution. Both statistics are zero for a Gaussian distribution but will depart from zero when the distribution is non-Gaussian. We can therefore estimate a "nonGaussian scale" $R_{\mathrm{NG}}$, below which both the skewness and the kurtosis depart significantly from zero.

Another useful statistic sensitive to the non-Gaussian features of a density field is the genus, which is employed to measure the topology of a continuous field. Conceptually, the genus of 


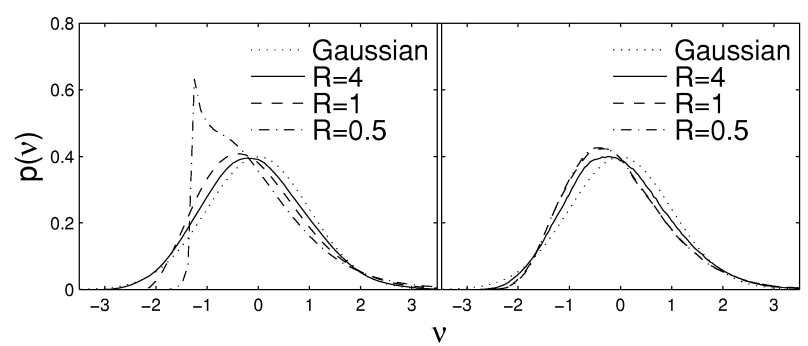

FIG. 1.-One-point PDF $p(\nu)$ of the density field in the linear string + CDM (left) and string + HDM (right) models for different $R$ [in $\left(\Omega h^{2}\right)^{-1} \mathrm{Mpc}$. Here $\sigma_{4 \mathrm{c}}: \sigma_{1 \mathrm{c}}: \sigma_{0.5 \mathrm{c}}: \sigma_{4 \mathrm{~h}}: \sigma_{1 \mathrm{~h}}: \sigma_{0.5 \mathrm{~h}} \approx 1: 5.2: 8.9: 0.69: 1.2: 1.3$. All simulation curves are averaged from six realizations, with standard deviation less then $5 \%$ of the amplitudes throughout.

a given surface can be defined as $g=$ number of holes number of isolated regions +1 . A more useful definition of genus is given by the Gauss-Bonnet theorem, which relates the integrated Gaussian curvature (a local property) of a surface with the genus (a global property) of that surface:

$$
\oint K d A=4 \pi(1-g),
$$

where $d A$ is a differential two-dimensional surface patch and $K=1 / a_{1} a_{2}$ is the Gaussian curvature of the patch with $a_{1}$ and $a_{2}$ the two principal radii of curvature. We used the numerical code developed by Avelino (1997) to calculate the genus of these isodensity surfaces, applying methods proposed by Gott, Mellot, \& Dickinson (1986). Here we employed the commonly used volume fraction parametrization of the genus curve, that is, we define a new variable $\nu_{f}$ so that

$$
f=\frac{1}{(2 \pi)^{1 / 2}} \int_{\nu_{f}}^{\infty} e^{-u^{2} / 2} d u,
$$

where $f$ is a previously calculated volume fraction of the whole simulation box within which the density is above a given threshold. Having done this, the genus curve becomes less sensitive to the PDF, which we studied separately.

\section{RESULTS}

In Figure 1 we plot the one-point PDF $p(\nu)$ of the density field in the linear string CDM and HDM models for different smoothing scales $R$. The skewness and kurtosis of $p(\nu)$ as functions of $R$ were plotted in Figure 2. Although on large scales string perturbations are almost indistinguishable from Gaussian random-phase perturbations, on scales smaller than $1.5\left(\Omega h^{2}\right)^{-1}$ Mpc the non-Gaussian character is remarkably distinct, especially in a CDM background.

In Figure 3 we plot the genus curves $g\left(\nu_{f}\right)$ for $R=$ $0.4\left(\Omega h^{2}\right)^{-1} \mathrm{Mpc}$ in both string $+\mathrm{CDM}$ and string + HDM models, as well as those of Gaussian random fields with correspondingly identical power spectra. These curves have been smoothed using three-point boxcar smoothing (see, for example, Vogeley et al. 1994) and were parametrized by the volume fraction (eq. [2]). We can observe that on these small scales, the genus curves of the cosmic string-seeded density fields deviate in a significant way from Gaussian random fields. As expected, the correlations among the phases of different Fourier modes imply a smaller number of independent regions in real space and therefore a smaller genus amplitude when

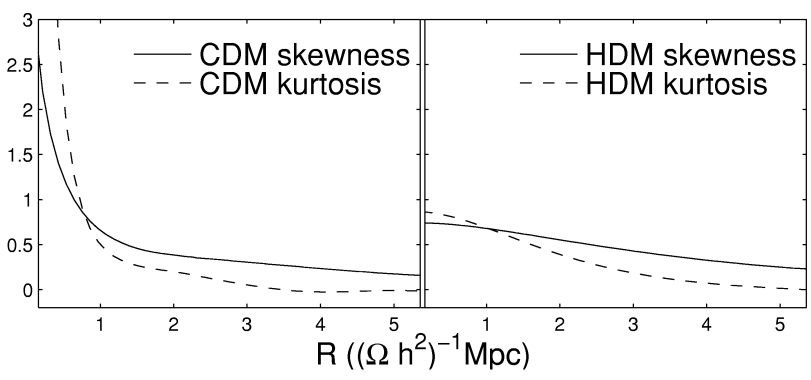

FIG. 2.-Skewness and kurtosis of $p(\nu)$ induced by cosmic strings.

compared with that of a Gaussian random field. This is illustrated in Figure 4, where two different isodensity thresholds for the CDM string model are directly compared with purely Gaussian fluctuations for $R=0.4\left(\Omega h^{2}\right)^{-1}$ Mpc. However, we have also verified that when smoothed on larger scales $R \gtrsim 3\left(\Omega h^{2}\right)^{-1} \mathrm{Mpc}$, cosmic string and Gaussian random-phase genus curves are very close to each other in both amplitude and shape. Finally, we note that the string + HDM model departure from a Gaussian distribution is less apparent than for string + CDM model throughout all of the analysis above.

\section{DISCUSSION}

In order to interpret our results, we invoked the semianalytic model described in Avelino et al. (1998a, 1998b; see also Albrecht \& Stebbins 1992). This model was shown to be very accurate in reproducing the simulation power spectrum in both matter-era and radiation-era scaling regimes, as well as during the radiation-era-matter-era transition. The power spectrum of string-induced density perturbations can be written as

$$
P(k)=16 \pi^{2} G^{2} \mu^{2} \int_{\eta_{i}}^{\eta_{f}}\left|\tilde{G}\left(k ; \eta_{0}, \eta\right)\right|^{2} \mathcal{F}(k, \eta) d \eta,
$$

where $\mu$ is the string energy density per unit length, $\tilde{\mathcal{G}}\left(k ; \eta_{0}, \eta\right)$ is the Fourier transform of the appropriate Green's functions, and $F(k, \eta)$ is the structure function. At a given time, $\mathcal{F}(k, \eta)$ has a turnover scale at $k_{\xi} \approx 20 / \eta$, which reflects the comoving correlation length of cosmic strings $\xi \approx \eta / 3$. At a particular time, the perturbations induced on scales larger than the string correlation length are generated by many string elements and, therefore, they are nearly Gaussian according to the central limit theorem. On the other hand, perturbations

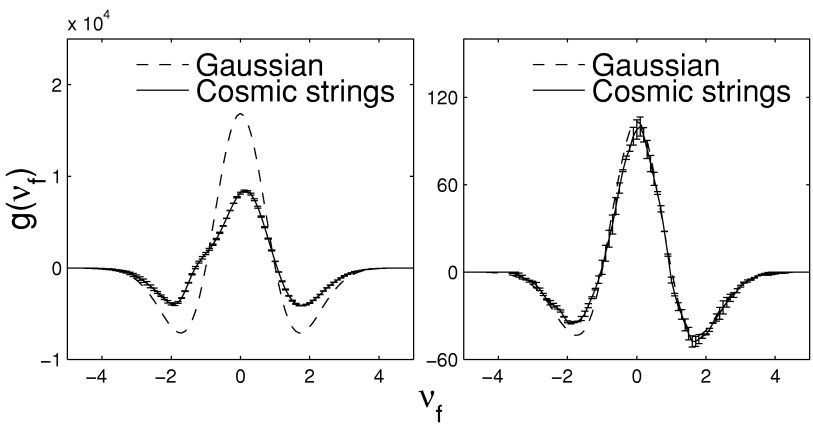

FIG. 3.-Genus curves in the CDM (left) and HDM (right) models. Here $R=0.4\left(\Omega h^{2}\right)^{-1} \mathrm{Mpc}$, and the box size is $\left(50 h^{-1} \mathrm{Mpc}\right)^{3}$. Error bars are taken from six realizations for each curve. 

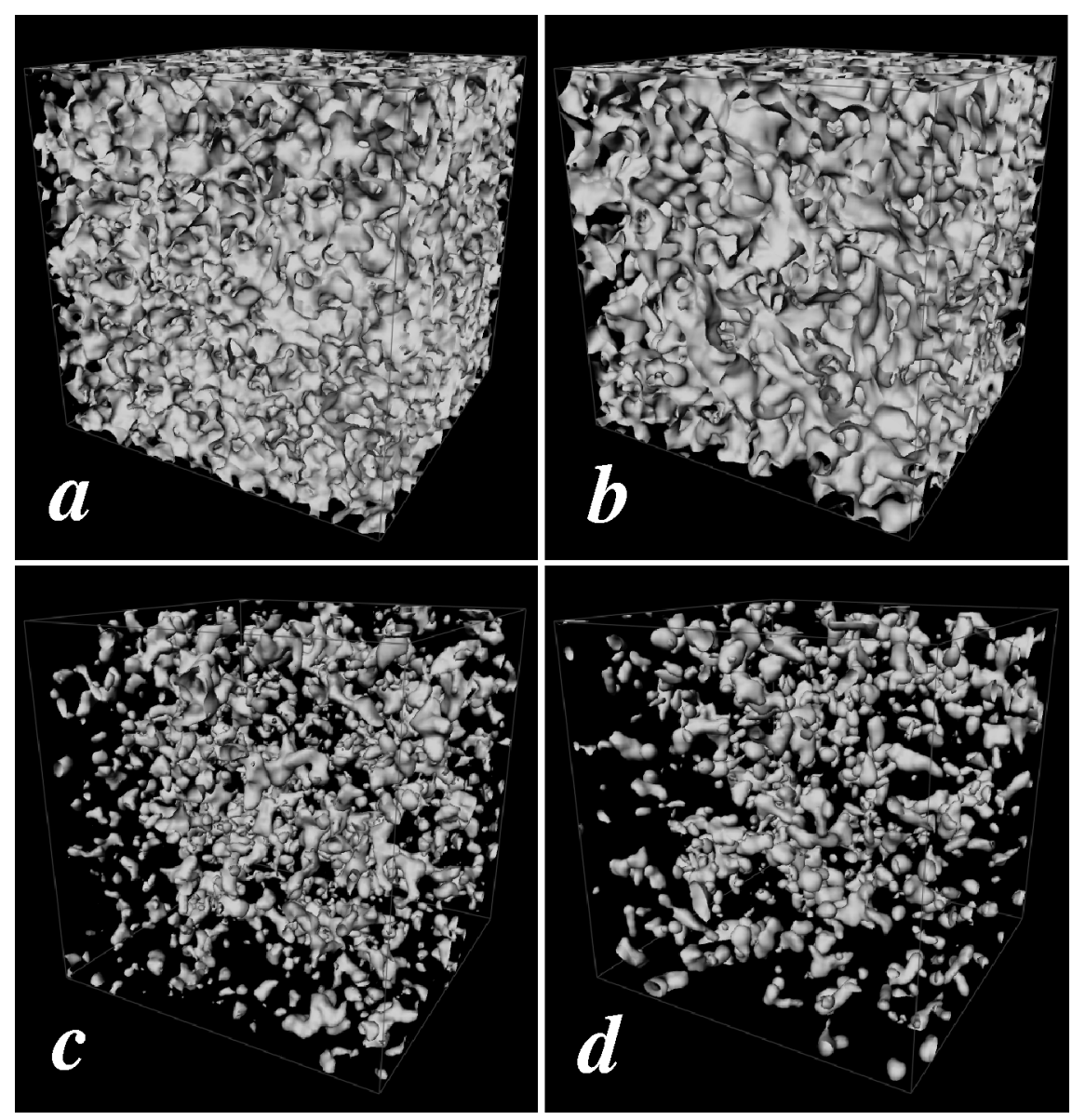

FIG. 4.-Isodensity surfaces with $(a, b) \nu_{f}=0$ and $(c, d) \nu_{f}=2$ for $(a, c)$ a Gaussian field and $(b, d)$ the string $+\mathrm{CDM}$ model. Here $R=0.4\left(\Omega h^{2}\right)^{-1} \mathrm{Mpc}$, and the box size is $\left(25 h^{-1} \mathrm{Mpc}\right)^{3}$.

induced on smaller scales are very non-Gaussian because they can be either very large within the string-induced wakes or else very small in regions outside these wakes. In consequence, we can roughly divide the power spectrum of cosmic string-seeded density perturbations into two parts: a nearly Gaussian part generated when the string correlation length was smaller than the scale under consideration and a strongly skewed non-Gaussian part generated when the string correlation length was larger. We shall abuse strict definitions and call these simply the "Gaussian" and "non-Gaussian" contributions, respectively. In terms of the structure function in equation (3), we can make the split $\mathcal{F}_{\mathrm{G}}(k, \eta)=\mathcal{F}(k, \eta)$ for $k<k_{\xi}\left(\mathcal{F}_{\mathrm{G}}=0\right.$ for $\left.k>k_{\xi}\right)$ and, similarly, $\mathcal{F}_{\mathrm{NG}}(k, \eta)=\mathcal{F}(k, \eta)$ for $k>k_{\xi}$. $\mathcal{F}_{\mathrm{G}}$ corre-

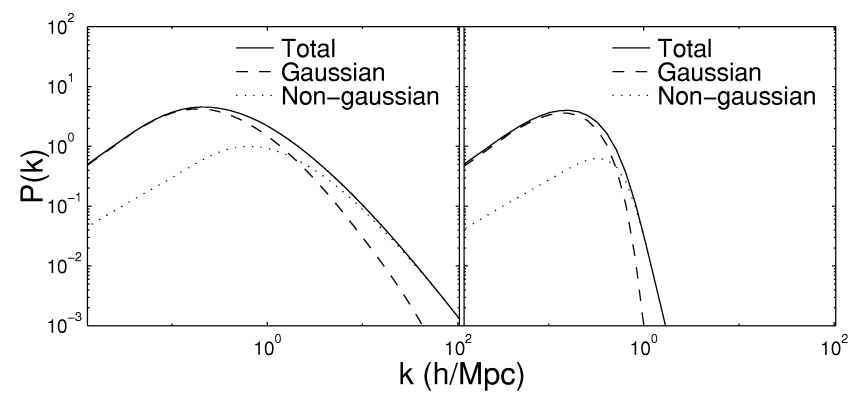

FIG. 5.-Estimated Gaussian and non-Gaussian contributions as a comparison with the total power spectrum in the CDM (left) and HDM (right) models. sponds to the left-hand side of the structure function from its peak to the causal compensation cutoff, where $F_{\mathrm{G}} \propto k^{4}$ for $k \ll k_{\xi}$. $F_{\mathrm{NG}}$ can be largely identified with the imprint of string wakes and behaves as $\mathcal{F}_{\mathrm{NG}} \propto k^{-2}$ for $k>k_{\xi}$.

Integrating equation (3) with this Gaussian/non-Gaussian split, we can compare the relative contributions to the total power spectrum in CDM and HDM models, as illustrated in Figure 5. This plot demonstrates that these two contributions become comparable at $k \sim 2 h \mathrm{Mpc}^{-1}$ for the CDM model and $k \sim 0.5 h \mathrm{Mpc}^{-1}$ for the HDM model, taking a Hubble parameter $H_{0}=70 \mathrm{~km} \mathrm{~s}^{-1} \mathrm{Mpc}^{-1}$. We further calculated the variance of the density fields from these non-Gaussian and Gaussian contributions when smoothed with a top-hat window of radius $R$ :

$$
\sigma_{R}^{2}=4 \pi \int_{0}^{\infty}|\tilde{w}(k R)|^{2} P(k) k^{2} d k
$$

where $\tilde{w}(x)=3 j_{1}(x) / x$ is the Fourier transform of a top-hat window and $j_{1}(x)$ the spherical Bessel function of order 1 . Figure 6 plots the ratio between the standard deviations of the non-Gaussian and Gaussian density perturbations $\sigma_{R(\mathrm{NG})} / \sigma_{R(\mathrm{G})}$ against $R$ for both CDM and HDM models. The criterion $\sigma_{R(\mathrm{NG})} / \sigma_{R(\mathrm{G})} \gtrsim 1$ gives an estimate for the non-Gaussian scale $R_{\mathrm{NG}}$. In Figure 6 , we also consider the effect of the limited dynamic range of our simulations by comparing with the full semianalytic integral (3). The simulations slightly enhance non- 


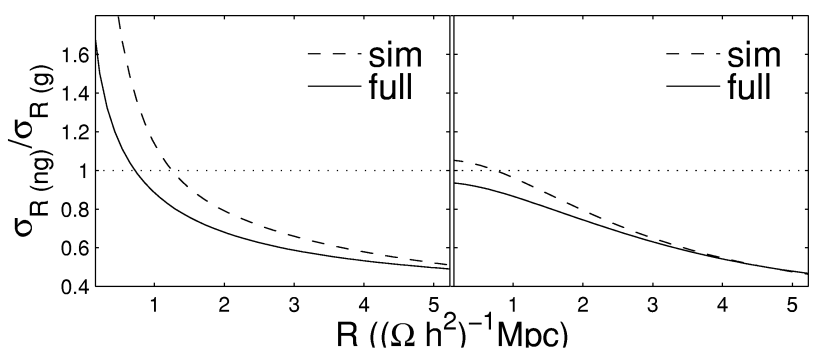

FIG. 6. - The ratio $\sigma_{R(\mathrm{NG})} / \sigma_{R(\mathrm{G})}$ against $R$ for both CDM (left) and $\mathrm{HDM}$ (right) models. The solid lines have a full dynamic range going from $\eta=0$ to today, while the dashed lines have the same dynamic range of our simulations.

Gaussianity essentially because there is more Gaussian power missing from early times $\eta<0.4 \eta_{\text {eq }}$. Nevertheless, we have checked that the power lost $\left[\sigma_{\text {(full) }}-\sigma_{\text {(sim) }}\right] / \sigma_{\text {(full }}$ on scales $R \geq 1\left(\Omega h^{2}\right)^{-1} \mathrm{Mpc}$ is always $\leq 10 \%$ for the non-Gaussian contribution, $\leq 35 \%$ for the Gaussian contribution, and $\leq 25 \%$ for the total power. This confirms the reliability of our non-Gaussian analysis based on these simulations.

The consistency between Figure 2 and Figure 6 should be evident, notably for the skewness. The physical reason for the much stronger non-Gaussian features on small scales can now be interpreted as the following. Before the radiation-matter equality $\eta_{\mathrm{eq}}$, the growth of density perturbations was suppressed. This means that even on small scales the contribution to the power spectrum from the matter era is very important. Adding the fact that by the time of $\eta_{\mathrm{ea}}$ the string correlation length had already grown to several $\left(\Omega h^{2}\right)^{-1} \mathrm{Mpc}$, the perturbations generated during the matter era on scales smaller than this will add an important non-Gaussian contribution to the total power spectrum. This also explains the peak in the PDF on small scales (see Fig. 1). The limited dynamic range of our simulations tends to produce a sharp peak on the PDF for small smoothing scales because of the presence of voids, that is, regions left largely unperturbed by the transition era strings. However, even for the full dynamic range a similar peak, although slightly smoother, should still emerge. This is the origin of the non-Gaussianity on smaller scales, the existence of which is not affected by the limited dynamic range, as we demonstrate in Figure 6. The reason that the HDM model is more Gaussian than the CDM model is that the neutrino free-streaming length prevents small-scale perturbations from emerging until late times. This significantly reduces the HDM power at large $k$, precisely where the non-Gaussian contribution is dominant in the CDM model (see Fig. 5). This also accounts for the smaller genus amplitude for the HDM model (see Fig. 3).

We also note that our results were obtained in the linear regime. When the density field evolves into the nonlinear regime at later times, the skewness increases as a direct result of the constraint that the density contrast $\delta \geq-1$. However, when compared with observations, the skewness of our linear cosmic string-seeded result for any reasonable value of $\Gamma$ is well below that of the Queen Mary and WestfieldDurham-Oxford-Toronto survey (Saunders et al. 1991). Our results are also consistent with those of Canavezes et al. (1997), since we find that cosmic string and Gaussian random-phase genus curves are very similar in the scale range in which no strong phase correlations were found by the genus analysis of the Point-Source Catalog redshift survey. For Gaussian models, it is well known that in the nonlinear regime the skewness is equal to its initial value plus a contribution due to the nonlinear collapse. This contribution scales proportionally to the rms density fluctuation $\sigma$ (Fry \& Scherrer 1994). Similarly, the nonlinear contribution to the kurtosis also has a simple scaling dependence on $\sigma$, depending on whether or not the initial density field is Gaussian (Chodorowski \& Bouchet 1995). This indicates that nonlinear clustering preserves the non-Gaussianity inherent in the linear evolution, but further investigation using $\mathrm{N}$-body simulations will be required to establish if the non-Gaussian signature of cosmic string models is potentially measurable.

\section{CONCLUSION}

We conclude that on length scales smaller than $1.5\left(\Omega h^{2}\right)^{-1}$ Mpc, perturbations seeded by cosmic strings are very nonGaussian, especially in the context of a CDM model. In an open or $\Lambda$ universe with $\Gamma=\Omega h \sim 0.15$, this scale will be shifted to $10 h^{-1} \mathrm{Mpc}$, which may still be in the linear or mildly nonlinear regime, thus potentially providing a strong empirical test for cosmic string models. It has been suggested that such non-Gaussianity may imply that it is difficult in string models to deduce the parameter $\beta=\Omega_{0}^{0.6} / \mathrm{b}$ from observations of density and velocity fields (van de Bruck 1998). However, our results indicate otherwise on large scales, since we find that string perturbations are very similar to Gaussian-random phase fluctuations when smoothed on sufficiently large scales.

We thank Carlos Martins and Robert Caldwell for useful conversations. P. P. A. is funded by JNICT (PRAXIS XXI/ BPD/9901/96). E. P. S. S. acknowledges support from PPARC grant GR/L 21488. J. H. P. W. is funded by CVCP (ORS/ 96009158) and by the Cambridge Overseas Trust (UK). B. A. acknowledges support from NSF grant PHY95-07740. This work was performed on COSMOS, the Origin2000 owned by the UK Computational Cosmology Consortium, supported by Silicon Graphics/Cray Research, HEFCE and PPARC.

\section{REFERENCES}

Albrecht, A., \& Robinson, J. 1995, preprint (astro-ph/9505123)

Albrecht, A., \& Stebbins, A. 1992, Phys. Rev. Lett., 68, 2121

Allen, B., \& Shellard, E. P. S. 1990, Phys. Rev. Lett., 64, 685

Avelino, P. P. 1997, ApJ, 487, 18

Avelino, P. P., Shellard, E. P. S., Wu, J. H. P., \& Allen, B. 1998a, Phys. Rev. Lett., 81, 2008 1998b, in preparation

Battye, R. A., Robinson, J., \& Albrecht, A. 1998, Phys. Rev. Lett., 80, 4847

Brandenberger, R. H., Kaplan, D. M., \& Ramsey, S. A. 1993, preprint (astro$\mathrm{ph} / 9310004)$

Canavezes, A., et al. 1997, preprint (astro-ph/9712228)

Chodorowski, M. J., \& Bouchet, F. R. 1995, preprint (astro-ph/9507038)

Fry, J. N., \& Scherrer, R. J. 1994, ApJ, 429, 36
Gott, J. R., Melott, A. L., \& Dickinson, M. 1986, ApJ, 306, 341 Moessner, R. 1995, MNRAS, 277, 927

Park, C., Spergel, D. N., \& Turok, N. 1991, ApJ, 372, L53

Peacock, J. A., \& Dodds, S. J. 1994, MNRAS, 267, 1020

Saunders, W., et al. 1991, Nature, 349, 32

Vachaspati, T. 1992, Phys. Lett. B, 282, 305

van de Bruck, C. V. 1998, Phys. Rev. D, 57, 4663

Viana, P. T. P., \& Liddle, A. R. 1996, MNRAS, 281, 323

Vilenkin, A., \& Shellard, E. P. S. 1994, Cosmic Strings and Other Topological Defects (Cambridge: Cambridge Univ. Press)

Vogeley, M. S., Park, C., Geller, M. J., Huchra, J. P., \& Gott, J. R. 1994, ApJ, 420,525 